\title{
A BAYESIAN BELIEF NETWORK APPROACH FOR PREDICTING KERNICTERUS
}

\author{
F. I. Amadin ${ }^{1, *}$ and M. E. Bello² \\ 1, 2, Department of Computer Science, University of Benin, Benin City, Edo StATE, NIGERIA \\ E-mail addresses: 1 frankamadin@uniben.edu, ${ }^{2}$ moseseromo@yahoo.com
}

\begin{abstract}
A lot of research have been conducted using expert systems in the diagnosis of neonatal jaundice but none has been conducted on kernicterus. Kernicterus is a complication of neonatal jaundice in which bilirubin accumulates in the grey matter of the brain, causing an irreversible neurological damage. In this paper, a Bayesian belief network was designed for predicting neonatal jaundice. The BBN model has 15 nodes and had $97 \%$ and $94 \%$ accuracy in classifying neonatal jaundice and kernicterus respectively.
\end{abstract}

Keywords: Jaundice, Kernicterus, Bayesian Belief Network

\section{INTRODUCTION}

Bilirubin is an orange-yellow pigment which is created by the breaking down of red blood cells by the liver. If the amount of bilirubin in the blood is high it results in jaundice $[1,2]$. Jaundice is a disease which is characterized by the yellowish color of the skin. This disease although common in new born babies can also affect both adults and children [3,4]. This is because their livers are not fully developed and it is incapable of effectively metabolizing the bilirubin for excretion [5]. Neonatal jaundice occurs in $65 \%$ of newborns and $80 \%$ of preterm neonates in tropical regions and within the first ten days of their lives [6, 7]. If this condition is not treated it results in kernicterus $[8,9]$. Kernicterus is a complication of neonatal jaundice in which bilirubin accumulates in the grey matter of the brain, causing an irreversible neurological damage [1]. Symptoms of kernicterus includes yellowish skin, difficulty or trouble sleeping, problem feeding and extreme fussiness at the early stage but manifestations of kernicterus symptoms as the child get older includes seizures, unusual motor development, muscle spasms, hearing and other sensory problem, inability to gaze up ward and stained tooth enamel [8]. Preterm have a high risk of developing neonatal jaundice and if not properly handled it can lead to kernicterus [8,9]. Diagnosis of neonatal jaundice has been a huge problem for medical experts [5]. Machine learning techniques have been applied in the area of medical diagnosis and has yielded excellent results $[5,10,11]$. In this paper, we aim to apply Bayesian Belief network in the prediction of kernicterus because of its capability to learn from noisy observations and also better at describing complex stochastic processes.

\section{RELATED WORKS}

A lot of research have been conducted using expert systems is the diagnosis of jaundice. In [10] an expert system for diagnosing neonatal jaundice was designed. The system was implemented using ASBRU language and it was able to save $25 \%$ cost on neonatal jaundice diagnosis. In [11] an expert system for diagnosing neonatal jaundice for medical practitioners in India was designed. The system was tested with data collected from various hospitals in India. The major short coming of the system is that it is incapable of learning from data. Similarly, in [5] a neuro-fuzzy based approach for diagnosing neonatal jaundice was proposed. The system was implemented using matlab. The system had two symptoms (bilirubin and weight) which was used in diagnosing neonatal jaundice. The Gaussian membership function were used in mapping values into fuzzy sets and the centroid technique was used in converting crisps value in the defuzzification layer. Results from the experiment indicated that the 
system has $80 \%$ accuracy in predicting neonatal jaundice.

\section{BAYESIAN BELIEF NETWORK}

Bayesian belief networks (BBN) are probabilistic network based models where nodes represents variables and edges shows the conditional dependency amongst variables. BBN is based on Bayes theorem which maps the cause-and-effect relationships between variables. In order to comprehend the connections in the BBN we considered the Bayes theorem by expressing conditional and marginal probabilities of two events a and $\beta$. This is shown in (1).

Where:

$$
P(\alpha \mid \beta)=\frac{P(\beta \mid \alpha) P(\alpha)}{P(\beta)}
$$

$P(a)$ is the probability of event a occurring without any information of event $\beta$

$P(\beta)$ is the probability of event $\beta$ occurring without any information of event a

$P(\beta \mid a)$ is the probability of event $\beta$ occurring given that event a has occurred

$P(a \mid \beta)$ is the probability of event a occurring given that event $\beta$ has occurred
The BBN model is an acyclic graph with nodes and edges which encodes the joint probability distribution for each variable on the network. The joint distribution for each node and the full joint distribution for the entire network is clearly described in (2) and (3) respectively.

$$
\begin{gathered}
P(A)=\prod_{i=1}^{n} P\left(A_{i} \mid \text { Parents }_{i}\right) \\
P\left(A_{1}, A_{2} \ldots . A_{n}\right)=\prod_{i=1}^{n} P\left(A_{i} \mid \text { Parents }_{i}\right)
\end{gathered}
$$

Where: $\mathrm{n}$ is the number of nodes in the BBN. Parent $A_{i}$ is the set of parent node of $A$

\section{DIAGNOSTIC MODEL}

The BBN structure of dependencies among the variables was elicited from domain experts at the University of Benin Teaching Hospital. The model for predicting kernicterus has 15 nodes which comprises of bilirubin level, yellowish skin, difficulty sleeping, age, fussiness, neonatal jaundice, drowsiness, weight, seizures, unusual motor development, muscle spasm, hearing and other sensory problem, inability to gaze up ward, stained tooth enamel and kernicterus. The BBN model is shown in Figure 1.

In Table 1 the probability of each leaf node in the BBN model is shown. The values were gotten from medical experts.

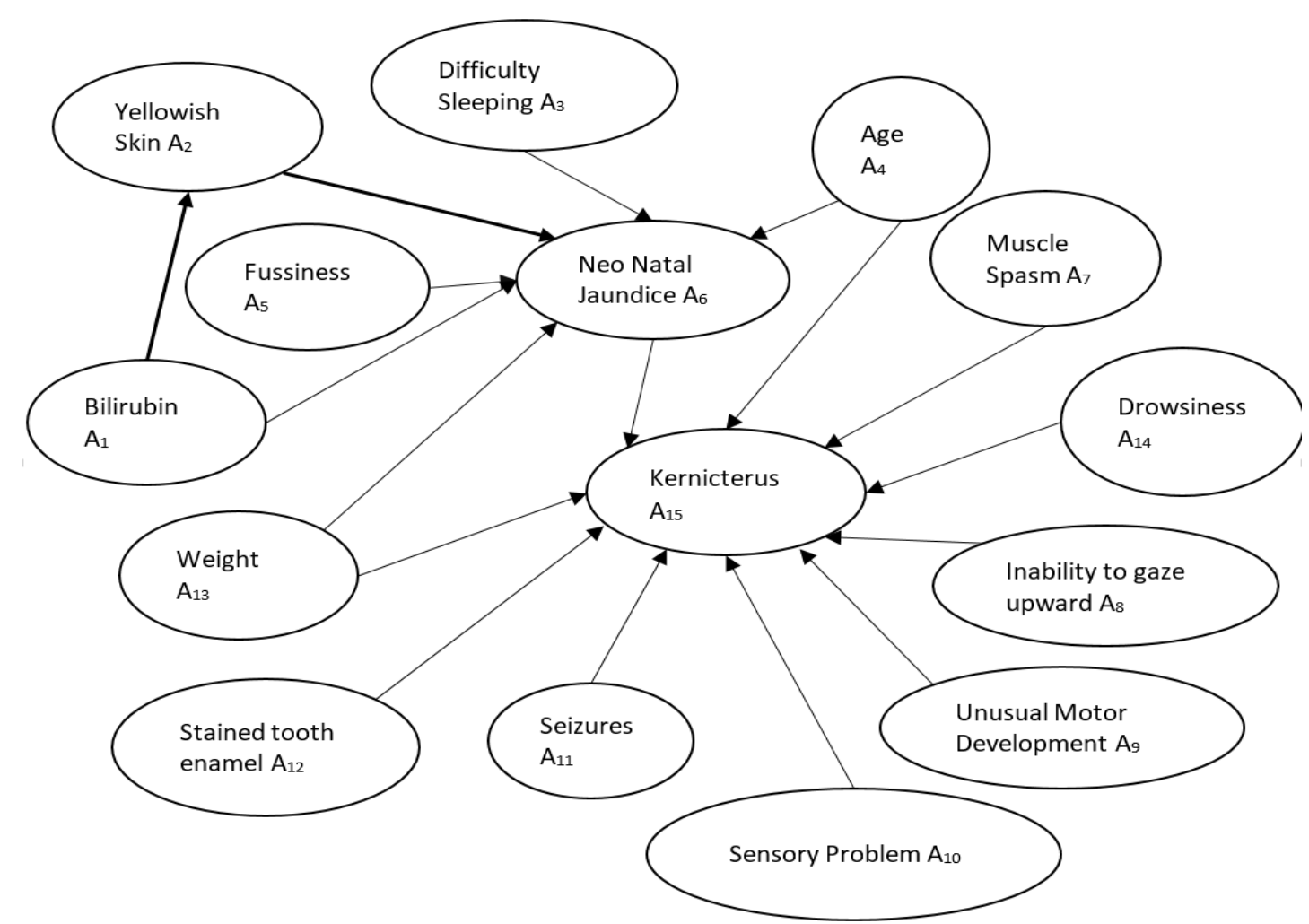

Figure 1: Bayesian Belief Network for Predicting Kernicterus 
Table 1: Distribution of Probability Values for Leaf Nodes in the BBN

\begin{tabular}{|c|c|c|}
\hline Node & Components & $\begin{array}{c}\text { Result (probability } \\
\text { value) }\end{array}$ \\
\hline \multirow{3}{*}{$\mathrm{A} 1$} & High & $85 \%$ \\
\hline & Normal & $10 \%$ \\
\hline & Low & $5 \%$ \\
\hline \multirow[b]{2}{*}{ A3 } & Present & $50 \%$ \\
\hline & Not Present & $50 \%$ \\
\hline \multirow{2}{*}{ A4 } & Present & $70 \%$ \\
\hline & Not Present & $30 \%$ \\
\hline \multirow{2}{*}{ A5 } & Present & $60 \%$ \\
\hline & Not Present & $40 \%$ \\
\hline \multirow{2}{*}{ A7 } & Present & $65 \%$ \\
\hline & Not Present & $35 \%$ \\
\hline \multirow{2}{*}{ A8 } & Present & $56 \%$ \\
\hline & Not Present & $44 \%$ \\
\hline \multirow{2}{*}{ A10 } & Present & $72 \%$ \\
\hline & Not Present & $38 \%$ \\
\hline \multirow{2}{*}{ A11 } & Present & $60 \%$ \\
\hline & Not Present & $40 \%$ \\
\hline \multirow{2}{*}{ A12 } & Present & $50 \%$ \\
\hline & Not Present & $50 \%$ \\
\hline \multirow{3}{*}{ A13 } & Over-Weight & $10 \%$ \\
\hline & Normal & $20 \%$ \\
\hline & Under-Weight & $70 \%$ \\
\hline \multirow{2}{*}{ A14 } & Present & $50 \%$ \\
\hline & Not Present & $50 \%$ \\
\hline
\end{tabular}

From Table 1 above, using the equivalence principle, the probability distributions for presence of bilirubin and yellowish skin are equivalent. Two symptoms are very important in predicting neonatal jaundice; level of bilirubin and age. Based on the Bayesian network model for predicting kernicterus in Figure 1 the probability of diagnosing a neonate with jaundice is given by the full joint distribution of node $A 6$ and this is shown in (4). The prediction of kernicterus is obtained by the joint probability distribution of node $A_{15}$ and this is shown in (5).

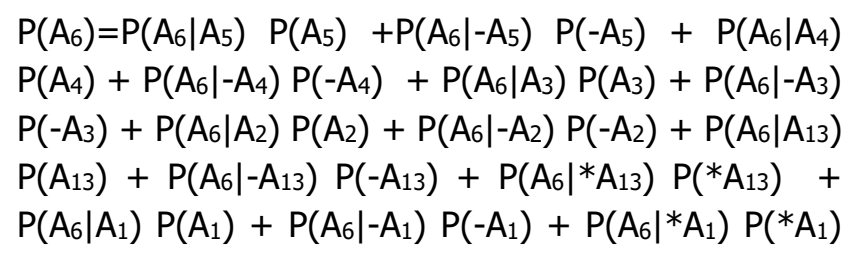

$$
-\mathrm{A}_{13}=\text { Normal, } \quad * \mathrm{~A}_{13}=\text { Underweight }
$$

$$
\begin{aligned}
P\left(A_{15}\right)= & P\left(A_{15} \mid A_{6}\right) P\left(A_{6}\right)+P\left(A_{15} \mid-A_{6}\right) P\left(-A_{6}\right)+P\left(A_{15} \mid A_{4}\right) \\
& P\left(A_{4}\right)+P\left(A_{15} \mid-A_{4}\right) P\left(-A_{4}\right)++P\left(A_{15} \mid A_{13}\right) P\left(A_{13}\right) \\
& +P\left(A_{15} \mid-A_{13}\right) P\left(-A_{13}\right)+P\left(A_{15} \mid * A_{13}\right) P\left(* A_{13}\right)+ \\
& P\left(A_{15} \mid A_{7}\right) P\left(A_{7}\right)+P\left(A_{15} \mid-A_{7}\right) P\left(-A_{7}\right)+ \\
& P\left(A_{15} \mid A_{14}\right) P\left(A_{14}\right)+P\left(A_{15} \mid-A_{14}\right) P\left(-A_{14}\right)+ \\
& P\left(A_{15} \mid A_{8}\right) P\left(A_{8}\right)+P\left(A_{15} \mid-A_{8}\right) P\left(-A_{8}\right)+ \\
& P\left(A_{15} \mid A_{9}\right) P\left(A_{9}\right)+P\left(A_{15} \mid-A_{9}\right) P\left(-A_{9}\right)++ \\
& P\left(A_{15} \mid A_{10}\right) P\left(A_{10}\right)+P\left(A_{15} \mid-A_{10}\right) P\left(-A_{10}\right)++ \\
& P\left(A_{15} \mid A_{11}\right) P\left(A_{11}\right)+P\left(A_{15} \mid-A_{11}\right) P\left(-A_{11}\right)++ \\
& P\left(A_{15} \mid A_{12}\right) P\left(A_{12}\right)+P\left(A_{15} \mid-A_{12}\right) P\left(-A_{12}\right)
\end{aligned}
$$

Where;

$A_{6}=$ Present,$\quad-A_{6}=$ Not Present,$\quad A_{7}=$ Present, $A_{7}=$ Not Present, $A_{14}=$ Present, $\quad-A_{14}=$ Not Present, $A_{4}=$ Infant, $\quad-A_{4}=$ Not Infant, $A_{8}=$ Present, $A_{8}=$ NotPresent, $A_{13}=$ Overweight, $-A_{13}=$ Normal, ${ }^{*} A_{13}=$ Underweight,$A_{9}=$ Present, $-A_{9}=$ Not Present, $A_{10}=$ Present,$\quad-A_{10}=$ Not Present, $\quad A_{11}=$ Present, $A_{11}=$ Not Present,$A_{12}=$ Present,$\quad-A_{12}=$ Not Present

\section{SIMULATION, RESULT AND DISCUSSION 5.1 Simulation and Result}

The BBN model was implemented using Bayes Server 7.5 and it had 15 nodes which consists of the symptoms (bilirubin level, yellowish skin, difficulty sleeping, age, fussiness, neonatal jaundice, drowsiness, weight, seizures, unusual motor development, muscle spasm, hearing and other sensory problem, inability to gaze up ward, stained tooth enamel and kernicterus) which could lead to kernicterus. The dataset used in training and testing the BBN model was gotten from the University of Benin Teaching Hospital (UBTH) and it contained 25 cases. A sample of the dataset is shown in Figure 2. The likelihood sampling algorithm was used in training the model. The likelihood function used is shown in the equation (6).

$$
\begin{aligned}
& \mathrm{L}(\theta: \mathrm{D})=\mathrm{P}(\mathrm{D} \mid \theta) \\
& =\prod_{1}^{\mathrm{N}} \mathrm{P}\left(\mathrm{S} 1^{\mathrm{N}}, \quad \mathrm{S} 2^{\mathrm{N}}, \quad \ldots \quad \mathrm{S} 15^{\mathrm{N}} \mid \theta\right)
\end{aligned}
$$

Where;

$\mathrm{D}=$ Dataset, $\mathrm{S} 1 \ldots \mathrm{S} 15=$ node on the network, $\mathrm{N}=$ number of parameter vector in the dataset,

$\theta=$ all parameter relevant for the calculation of the probability of a given node.

The BBN was trained using 10 known cases while the remainder was used in testing the model. The training 
dataset was arranged in the form as shown in equation (7).

$$
\mathrm{D}=\left[\begin{array}{c}
\mathrm{X}^{1} \\
\mathrm{X}^{2} \\
\cdot \\
\mathrm{X}^{\mathrm{N}}
\end{array}\right]=\left[\begin{array}{cccc}
\mathrm{S} 1^{1} & \mathrm{~S}^{1} & \ldots & \mathrm{S} 15^{1} \\
\mathrm{~S} 1^{2} & \mathrm{~S}^{2} & \ldots & \mathrm{S} 1^{2} \\
\cdot & \cdot & & \cdot \\
\mathrm{S}^{\mathrm{N}} & \mathrm{S2}^{\mathrm{N}} & \ldots & \mathrm{S} 15^{\mathrm{N}}
\end{array}\right]
$$

Where;

$\mathrm{D}=$ dataset, $\mathrm{X}^{\mathrm{i}}=$ parameter vector $\mathrm{i}, \mathrm{S} 1$...S15 = node on the network $\mathrm{N}=$ number of parameter vector in the dataset.

Each data in the dataset contains an outcome for neonatal jaundice and kernicterus. During testing the BBN model computes the joint probability distribution for kernicterus and neonatal jaundice and compares the results with the outcome in the dataset.

The results obtained from the Bayes Server showed that the BBN model used for predicting kernicterus had a prediction accuracy of $97 \%$ for neonatal jaundice and $95 \%$ for kernicterus. The results generated from the Bayes server are shown in the figures below. Figure 3, Figure 4, Figure 5 and Figure 6 show the BBN model in Bayes Server, loglikelihood of the dataset, prediction graph loglikelihood and prediction of neonatal jaundice (A6) and kernicterus (A15) respectively.

\begin{tabular}{|c|c|c|c|c|c|c|c|c|c|c|c|c|}
\hline BILILUBRIN & YELLOWISH & DIFFICULTY & AGE & FUSSINESS & MUSCLE SPASM & GAZING & MOTOR & SENSORY & SEIZURES & STAINED & WEIGHT & DROWSINESS \\
\hline 0.52 & 0.168 & 0.0851 & 0.0891 & 0.0955 & 0.763 & 0.392 & 0.822 & 0.0584 & 0.58 & 0.0521 & 0.17 & 0.348 \\
\hline 0.722 & 0.334 & 0.0553 & 0.424 & 0.343 & 0.0507 & 0.739 & 0.0479 & 0.0782 & 0.399 & 0.839 & 0.032 & 0.632 \\
\hline 0.247 & 0.29 & 0.357 & 0.58 & 0.332 & 0.598 & 0.471 & 0.262 & 0.00992 & 0.633 & 0.544 & 0.628 & 0.982 \\
\hline 0.736 & 0.246 & 0.409 & 0.596 & 0.354 & 0.95 & 0.462 & 0.859 & 0.0556 & 0.631 & 0.633 & 0.588 & 0.685 \\
\hline 0.638 & 0.666 & 0.00541 & 0.391 & 0.857 & 0.26 & 0.0406 & 0.659 & 0.067 & 0.53 & 0.876 & 0.347 & 0.0665 \\
\hline 0.976 & 0.665 & 0.942 & 0.428 & 0.089 & 0.46 & 0.145 & 0.892 & 0.0817 & 0.314 & 0.24 & 0.752 & 0.954 \\
\hline 0.808 & 0.107 & 0.414 & 0.372 & 0.601 & 0.975 & 0.147 & 0.753 & 0.0885 & 0.396 & 0.594 & 0.394 & 0.103 \\
\hline 0.743 & 0.877 & 0.473 & 0.512 & 0.673 & 0.688 & 0.557 & 0.791 & 0.0798 & 0.947 & 0.533 & 0.549 & 0.65 \\
\hline 0.942 & 0.972 & 0.13 & 0.671 & 0.859 & 0.619 & 0.0734 & 0.765 & 0.0566 & 0.713 & 0.79 & 0.624 & 0.694 \\
\hline 0.408 & 0.361 & 0.856 & 0.964 & 0.762 & 0.361 & 0.354 & 0.358 & 0.0882 & 0.509 & 0.217 & 0.504 & 0.386 \\
\hline 0.254 & 0.115 & 0.561 & 0.000237 & 0.91 & 0.776 & 0.532 & 0.633 & 0.0471 & 0.934 & 0.899 & 0.464 & 0.19 \\
\hline 0.724 & 0.993 & 0.99 & 0.937 & 0.66 & 0.252 & 0.68 & 0.902 & 0.0246 & 0.726 & 0.619 & 0.836 & 0.0867 \\
\hline 0.954 & 0.741 & 0.0202 & 0.406 & 0.548 & 0.911 & 0.05 & 0.0034 & 0.0441 & 0.73 & 0.254 & 0.183 & 0.929 \\
\hline 0.169 & 0.973 & 0.0361 & 0.204 & 0.509 & 0.95 & 0.537 & 0.146 & 0.0549 & 0.764 & 0.541 & 0.879 & 0.119 \\
\hline 0.912 & 0.591 & 0.34 & 0.475 & 0.722 & 0.345 & 0.711 & 0.444 & 0.0861 & 0.601 & 0.267 & 0.283 & 0.098 \\
\hline 0.249 & 0.508 & 0.438 & 0.223 & 0.771 & 0.57 & 0.31 & 0.0444 & 0.00204 & 0.136 & 0.954 & 0.329 & 0.24 \\
\hline 0.048 & 0.798 & 0.0929 & 0.654 & 0.367 & 0.535 & 0.383 & 0.763 & 0.0446 & 0.778 & 0.559 & 0.704 & 0.634 \\
\hline 0.73 & 0.0454 & 0.977 & 0.886 & 0.873 & 0.322 & 0.616 & 0.868 & 0.0299 & 0.374 & 0.62 & 0.101 & 0.306 \\
\hline 0.236 & 0.705 & 0.393 & 0.0926 & 0.567 & 0.65 & 0.222 & 0.179 & 0.0099 & 0.0557 & 0.426 & 0.749 & 0.884 \\
\hline 0.412 & 0.147 & 0.0753 & 0.018 & 0.0651 & 0.854 & 0.703 & 0.816 & 0.0669 & 0.797 & 0.633 & 0.75 & 0.676 \\
\hline 0.0993 & 0.271 & 0.283 & 0.0731 & 0.367 & 0.773 & 0.305 & 0.155 & 0.0873 & 0.693 & 0.0952 & 0.864 & 0.131 \\
\hline 0.666 & 0.433 & 0.399 & 0.813 & 0.384 & 0.574 & 0.146 & 0.169 & 0.00765 & 0.637 & 0.93 & 0.861 & 0.635 \\
\hline 0.866 & 0.326 & 0.143 & 0.652 & 0.0967 & 0.566 & 0.819 & 0.187 & 0.0711 & 0.724 & 0.95 & 0.584 & 0.43 \\
\hline 0.865 & 0.0745 & 0.434 & 0.736 & 0.522 & 0.414 & 0.263 & 0.642 & 0.087 & 0.827 & 0.754 & 0.296 & 0.656 \\
\hline 0.228 & 8 & 0.125 & 10.662 & 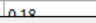 & -0.222 & 1065 & & كتعمة & ה הסم & & 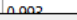 & \\
\hline
\end{tabular}

Figure 2: Dataset

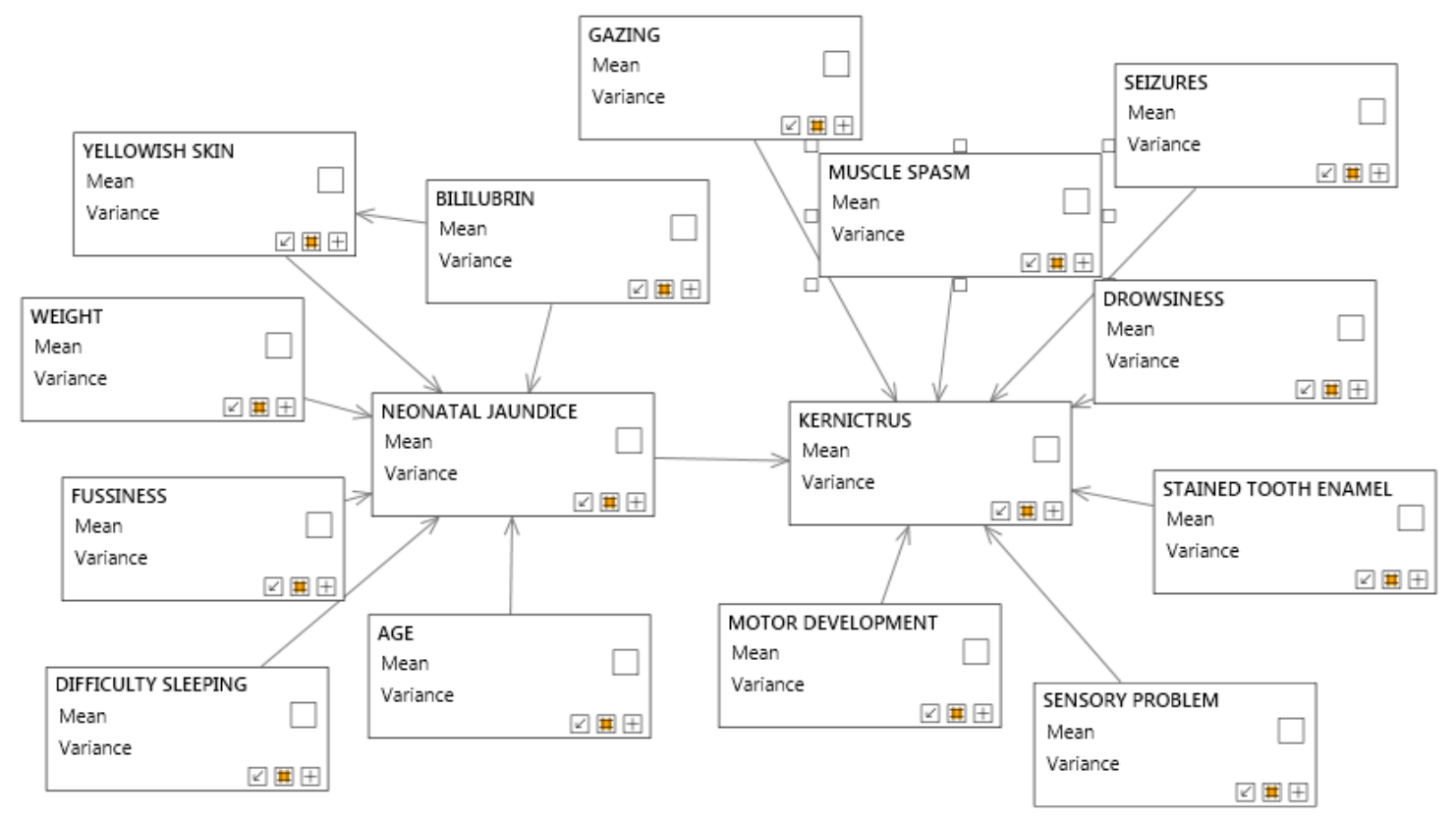

Figure 3: Bayesian Belief Network Model for Predicting Kernicterus 


\subsection{Discussion}

A lot of research have been conducted using expert systems is the diagnosis of neonatal jaundice but none has been conducted on kernicterus. In our paper we utilized a Bayesian belief network in predicting kernicterus and neonatal jaundice. Our system differs considerably from the ones proposed in [9] and [10] because those proposed in them are rule based systems and are incapable of learning. Our work is similar to the experiment conducted in [5] where neuro fuzzy system was used in predicting neonatal jaundice .Bilirubin and weight were the symptoms used and the system had a diagnostic accuracy of $80 \%$. In our work we used 5 symptoms in predicting neonatal jaundice and we had a prediction accuracy of
$97 \%$ which is way more accurate than the neuro-fuzzy system proposed in [5]. Also our system extended the functionality of the system proposed in [5] by being able to predict kernicterus in neonates.

\section{CONCLUSION}

Kernicterus is a complication of neonatal jaundice in which bilirubin accumulates in the grey matter of the brain, causing an irreversible neurological damage. In this paper, a Bayesian Belief Network BBN was designed for predicting neonatal jaundice. The BBN model had 15 nodes and had $97 \%$ and $94 \%$ accuracy in classifying neonatal jaundice and kernicterus respectively.

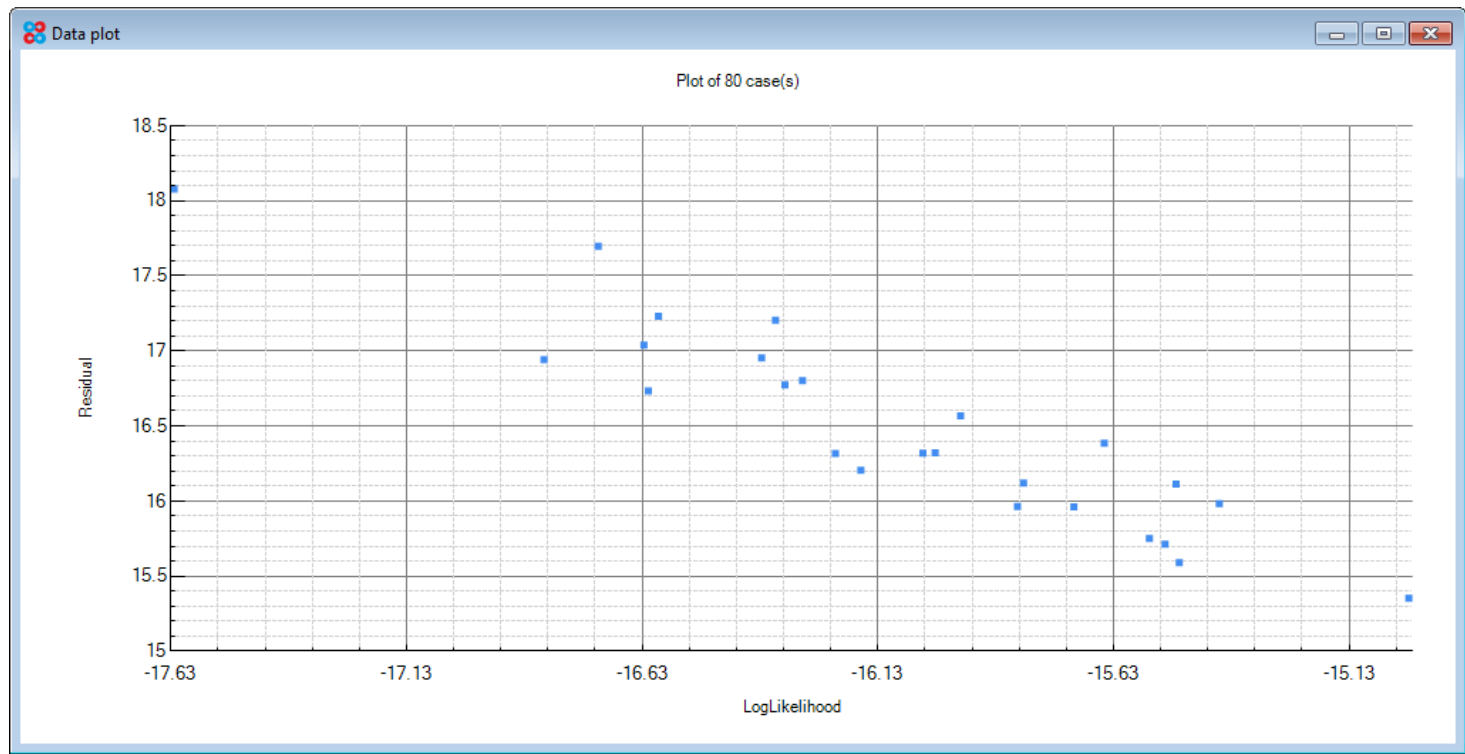

Figure 4: Log Likelihood of the Dataset

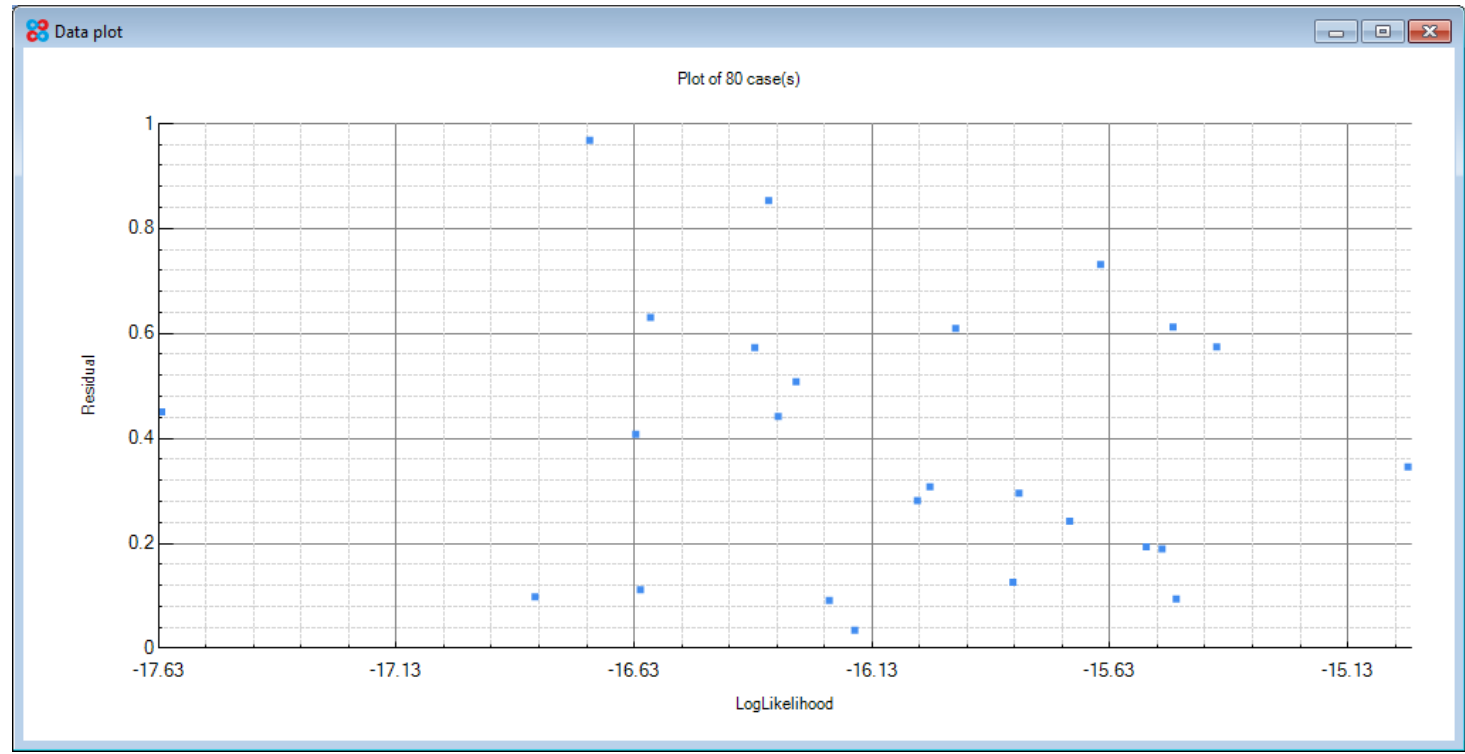

Figure 5: Prediction Graph Likelihood against Log Likelihood 


\begin{tabular}{|c|c|c|c|}
\hline LogLikelihood & Likelihood & Predict(NEONATAL & Predict(KERNICTRUS) \\
\hline-15 & $3.04 \mathrm{E}-07$ & 0.317 & 0.346 \\
\hline-15.7 & $1.59 \mathrm{E}-07$ & 0.671 & 0.731 \\
\hline-15.8 & $1.34 \mathrm{E}-07$ & 0.758 & 0.296 \\
\hline-16.2 & $9.49 \mathrm{E}-08$ & 0.227 & 0.0347 \\
\hline-15.6 & $1.75 \mathrm{E}-07$ & 0.302 & 0.194 \\
\hline-16.6 & $6.18 \mathrm{E}-08$ & 0.304 & 0.631 \\
\hline-16 & $1.17 \mathrm{E}-07$ & 0.648 & 0.609 \\
\hline-16.6 & $6.05 E-08$ & 0.286 & 0.112 \\
\hline-16.8 & $4.84 \mathrm{E}-08$ & 0.297 & 0.0981 \\
\hline-16 & $1.08 \mathrm{E}-07$ & 0.889 & 0.281 \\
\hline-16.2 & $8.99 \mathrm{E}-08$ & 0.656 & 0.0914 \\
\hline-17.6 & $2.21 \mathrm{E}-08$ & 0.849 & 0.451 \\
\hline-16.4 & $7.92 \mathrm{E}-08$ & 0.393 & 0.853 \\
\hline-16.3 & 8.08E-08 & 0.862 & 0.442 \\
\hline-15.5 & $1.87 \mathrm{E}-07$ & 0.134 & 0.0945 \\
\hline-15.5 & $1.85 \mathrm{E}-07$ & 0.622 & 0.612 \\
\hline-15.8 & $1.32 \mathrm{E}-07$ & 0.221 & 0.126 \\
\hline-16.6 & $5.99 \mathrm{E}-08$ & 0.79 & 0.408 \\
\hline-15.7 & $1.49 \mathrm{E}-07$ & 0.861 & 0.242 \\
\hline-16.4 & $7.69 \mathrm{E}-08$ & 0.847 & 0.573 \\
\hline-15.4 & $2.03 \mathrm{E}-07$ & 0.802 & 0.574 \\
\hline-16.7 & $5.44 \mathrm{E}-08$ & 0.746 & 0.968 \\
\hline-16 & $1.11 \mathrm{E}-07$ & 0.258 & 0.308 \\
\hline-16.3 & 8.39E-08 & 0.759 & 0.508 \\
\hline
\end{tabular}

Figure 6: Prediction of Neonatal Jaundice $\left(A_{6}\right)$ and Kernicterus $\left(A_{15}\right)$

\section{REFERENCES}

[1] Hameed N. N., Na'Ma A. M., Vilms R. and Bhutani V. K. "Severe neonatal hyperbilirubinaemia and adverse short-term consequences in Baghdad, Iraq. Neonatology 2011;100:57-63.

[2] Ogunlesi T. A., Dedeke I. O., Adekanmbi A. F., Fetuga M. B., and Ogunfowora O. B. "The incidence and outcome of bilirubin encephalopathy in Nigeria: a bi-centre study". Niger J Med;16:3549.

[3] Ojukwu J. U. and Ogbu C. N. Analysis and outcome of admissions in the special care baby unit of the Ebonyi State University Teaching Hospital, Abakaliki. J Coll Med; 2004.9: 2, 93-96.

[4] Okechukwu A, and Achonwa A. Morbidity and mortality patterns of admissions into the special care baby unit of University of Abuja Teaching Hospital. Niger J Clin Pract ;4:389-94.

[5] Mohammad S, Kamran K, K., Behrooz M., Nasser S. and Ali R. "A Neuro-Fuzzy Approach to Diagnosis of Neonatal Jaundice" Proceedings of the 1st international conference on Bioinspired models of network, information and computing systems, 2006, 1(16).
[6] Nelson, W. E. Behrman, R. E. Jenson, H. B. and Nelson R. M. (2000) "Textbook of Pediatrics," 16th Edition, New Jersey: W.B Saunders Co., pp. 515519, 2000.

[7] Mwaniki M. K, Gatakaa H. W., and Mturi F. N., An increase in the burden of neonatal admissions to a rural district hospital in Kenya over 19 years. BMC Public Health ;10:591, 2010.

[8] Olusanya B. O., Ogunlesi T. A. and Slusher T. M. "Why is kernicterus still a major cause of deathand disability in low-income and middle-income countries?" Arch Dis Child 99:1117-1121, 2014

[9] Omoigberale A. I, Sadoh W. E., Nwaneri D. U. "A 4 Year Review of Neonatal Outcome at the University of Benin Teaching Hospital, Benin City." Niger J Clin Pract; 13:321-325, 2010.

[10] Seyfang, S. Miksch, M. Marcos, "Combining Diagnosis and Treatment Using ASBRUE", 10th World Congress on Medical Informatics, Austria, 2001.

[11] Dharmar C., Srinivasan S., Mital D, and Haque S. "Expert system for the diagnosis of neonatal jaundice for use by medical field personnel" International Conference on Control, Automation, Robotics and Vision, 2002. 